\title{
Leishmania major Self-Limited Infection Increases Blood Cholesterol and Promotes Atherosclerosis Development
}

\author{
Luciana R. Fernandes, ${ }^{1}$ Ana Cecília C. Ribeiro, ${ }^{1}$ Marcela Segatto, ${ }^{1}$ Luís Felipe F. F. Santos, \\ Joana Amaral, ${ }^{2}$ Luciane R. Portugal, ${ }^{1}$ and Jacqueline I. A. Leite ${ }^{1}$ \\ ${ }^{1}$ Departamento de Bioquímica e Imunologia, Universidade Federal Minas Gerais, 31270-901 Belo Horizonte, MG, Brazil \\ ${ }^{2}$ Centro Universitário, Av. Prof. Mário Werneck, 1685-Estoril, 30455-610 Belo Horizonte, MG, Brazil \\ Correspondence should be addressed to Jacqueline I. A. Leite; jalvarezleite@gmail.com
}

Received 6 February 2013; Revised 30 March 2013; Accepted 31 March 2013

Academic Editor: Gloria L. Vega

Copyright (C) 2013 Luciana R. Fernandes et al. This is an open access article distributed under the Creative Commons Attribution License, which permits unrestricted use, distribution, and reproduction in any medium, provided the original work is properly cited.

\begin{abstract}
Leishmania major infection of resistant mice causes a self-limited lesion characterized by macrophage activation and a Th1 proinflammatory response. Atherosclerosis is an inflammatory disease involving hypercholesterolemia and macrophage activation. In this study, we evaluated the influence of L. major infection on the development of atherosclerosis using atherosclerosissusceptible apolipoprotein E-deficient (apoE KO) mice. After 6 weeks of infection, apoE KO mice exhibited reduced footpad swelling and parasitemia similar to C57BL/6 controls, confirming that both strains are resistant to infection with L. major. L. majorinfected mice had increased plasma cholesterol levels and reduced triacylglycerols. With regard to atherosclerosis, noninfected mice developed only fatty streak lesions, while the infected mice presented with advanced lesions containing a necrotic core and an abundant inflammatory infiltrate. CD36 expression was increased in the aortic valve of the infected mice, indicating increased macrophage activation. In conclusion, L. major infection, although localized and self-limited in resistant apoE KO mice, has a detrimental effect on the blood lipid profile, increases the inflammatory cell migration to atherosclerotic lesions, and promotes atherogenesis. These effects are consequences of the stimulation of the immune system by L. major, which promotes the inflammatory components of atherosclerosis, which are primarily the parasite-activated macrophages.
\end{abstract}

\section{Introduction}

Leishmania major is a protozoan parasite transmitted by sandflies of the genus Lutzomyia that inject the promastigote form into the dermis of the host. Once injected, the parasite is rapidly enclosed by phagocytic cells and transforms into the replicative intracellular amastigote form [1]. In immunocompetent hosts, such as C57BL/6 mice, L. major infection is a self-contained cutaneous lesion that elicits a Th1 immune response. In infected mice, the immune cells (macrophages, dendritic cells, natural killer cells, and $\mathrm{T}$ cells) produce cytokines and bioactive molecules, such as IFN- $\gamma$, IL-12, and nitric oxide (NO), which act against the protozoan [1].

Atherosclerosis is a chronic inflammatory disease associated with a high level of total cholesterol and proatherogenic lipoproteins (VLDL, IDL, and LDL), a prothrombotic status, and a Th1-polarized immune response [2, 3]. Macrophage and endothelial cell activation by atypical lipoproteins and proinflammatory cytokines induces the production of adhesion molecules, cytokines and chemokines, and causes oxidative stress [4]. Among atherosclerosis risk factors, infection plays an important role in the perpetuation of chronic inflammation at the atherosclerotic site $[5,6]$. Several studies suggest that pathogens, such as bacteria, viruses, and protozoa, contribute to the atherogenesis process [7-11]. The increased inflammation caused by these pathogens promotes macrophage activation and migration to the atheroprone site. Alternatively, proatherogenic status may be attributed to the systemic oxidative stress induced by infection, which enhances lipoprotein or endothelium oxidation. Pathogens involved in atherosclerosis development usually induce a systemic infection instead of a localized infection such as 
an L. major infection. However, even localized infections, such as odontologic ones, may be associated with the development of atherosclerosis [12].

Apolipoprotein (apo) E is a component of chylomicron and very low-density lipoproteins (VLDL) and mediates the uptake of these lipoproteins and possesses anti-inflammatory and antioxidant effects [13]. ApoE KO mice have impaired clearance of VLDL and chylomicrons from the blood, which results in hypercholesterolemia and favors the development of atherosclerotic lesions [11]. ApoE also has antiinflammatory and antioxidative properties, and its absence is partially responsible for the higher inflammatory profile of apoE-deficient animals compared to the wild-type control, C57BL/6 mice [14]. ApoE KO mice spontaneously develop atherosclerosis even when fed a normolipidemic diet [15]. The atherosclerotic lesions of apoE KO mice exhibit a similar distribution, microscopic appearance, and cellular composition to those found in humans. The atherosclerotic lesions are characterized by endothelial dysfunction, macrophage, and $\mathrm{T}$ cell infiltration and production of proinflammatory cytokines, such as TNF- $\alpha$, INF- $\gamma$, and IL-6, which indicates a typical Th1 polarized immune response [2].

This work aimed to determine if a self-limited L. major infection would be sufficient to modify the lipid metabolism and promote the development of atherosclerosis similar to what occurs with systemic infections.

\section{Material and Methods}

This protocol was approved by the Animal Care Committee of Universidade Federal de Minas Gerais (CETEA \#147/05).

2.1. Mice and Diet. Twenty-eight 11-week-old female apoE KO mice were separated into control (noninfected) and Leishmania major (L. major) infected groups. For the background control, twenty wild-type C57BL/6 mice were divided into control (noninfected) or L. major infected groups. Animals of both strains were equally distributed according to weight and blood cholesterol and were fed on AIN-93G standard diet [15].

2.2. Leishmania major Infection. The mice were infected with L. major (clone WHO MHOM/IL/80/Friedlin). The inoculum was prepared under sterile conditions from five-day $L$. major cultures, which corresponds to the stationary growth phase rich in the promastigote metacyclic infectious forms. The medium containing L. major was centrifuged, and the pellet was resuspended in $1 \mathrm{~mL}$ of PBS. Approximately $1 \times 10^{6}$ parasites were inoculated into the left footpad, and the right footpad was used as a control after inoculation with PBS as previously described [16]. The footpad was measured weekly with a caliper, and the amount of swelling was calculated as the difference between the measures of the infected (left) and control (right) footpads.

The animals' body weight and food intake were measured weekly for 6 weeks after infection. All groups received the same amount of food to avoid confounding the experiment with different amounts of nutrients. After 6 weeks and an overnight fast, the mice were euthanized under anesthesia. Plasma, footpads, and tissues were collected for subsequent analyses. The infected footpad was weighed without the skin and used for parasite quantification as previously described [16].

2.3. Lipids Assay. The total cholesterol, cholesterol in high density lipoprotein (HDLc) form, and triacylglycerols in the plasma were measured using commercial kits (Doles, Brazil) as previously described [17]. The amount of non-HDLc (that represents the atherogenic fractions VLDLc and IDLc) in the plasma was determined by calculating the difference between the total cholesterol and the HDLc.

The liver was washed in PBS and dried on filter paper. The contents of the cecum were separated from the lumen. The hepatic and cecal lipid extractions were performed as previously described by Folch et al. [18].

2.4. Histological Analysis. For the analysis of atherosclerosis, the aortic valves were washed with PBS, gently perfused with $10 \%$ neutral buffered formalin, and embedded in paraffin. The analysis was performed on 10 samples per group. The specimens were fixed in $3.7 \%$ paraformaldehyde and processed in paraffin. Briefly, every consecutive section $(10 \mu \mathrm{m}$ thick) through the aortic valve $(300 \mu \mathrm{m})$ was taken and stained with hematoxylin and eosin [19]. The sections used for immunofluorescence were deparaffinized by 2 xylene washes ( $30 \mathrm{~min}$ ) and then transferred to 3 washes (15 minutes each) of ethanol at concentrations of $100 \%, 85 \%$, and $70 \%$. The atherosclerotic lesion area was quantified using the Image Pro Plus software (Media Cybernetics, USA) from the sum of the atherosclerotic lesion areas obtained from the selected 10 sections, spaced $20 \mu \mathrm{m}$ apart, as previously described [11]. The inflammatory cells per field were counted automatically by the Image Pro Plus software ( $n=5$ mice, 3 nonconsecutive sections per animal) [11].

2.5. L. major Parasitism. The DNA in liver and spleen was extracted with proteinase K (Qiagen USA) according to the manufacturer's instructions. A PCR analysis was conducted [20]. Briefly, $2 \mu \mathrm{L}$ of DNA sample was added to $1.2 \mu \mathrm{L}$ of Taq polymerase buffer, $1.0 \mu \mathrm{L}$ of dNTPs $(2.5 \mathrm{mM}), 1.0 \mu \mathrm{L}$ of primers, $0.05 \mu \mathrm{L}$ of Taq polymerase, and $5.75 \mu \mathrm{L}$ of Milli$\mathrm{Q}$ sterile $\mathrm{H}_{2} \mathrm{O}$. The samples were placed in a thermocycler and the amplification conditions were as follows (35 cycles): denaturation at $94^{\circ} \mathrm{C}$ for $1 \mathrm{~min}$, annealing at $54^{\circ} \mathrm{C}$ for $1 \mathrm{~min}$, elongation at $72^{\circ} \mathrm{C}$ for $1.5 \mathrm{~min}$, and extension at $72^{\circ} \mathrm{C}$ for $10 \mathrm{~min}$. The PCR products were then subjected to gel electrophoresis polyacrylamide and visualized by silver staining. The sequences of the primers used, which correspond to the minicircle kDNA [20], are Uni 21$5^{\prime}$ GGGGTTGGTGTAAAATAAGGCC $3^{\prime}$ and LmJ4-5'CTA.

2.6. Immunofluorescence Staining. Cross-sections of the aortic roots were blocked with $4 \%$ (wt/vol) BSA in PBS. Next, sections were incubated for $1 \mathrm{~h}$ at $22^{\circ} \mathrm{C}$ with FITC-goat antibody to mouse CD11b $(0.2 \mathrm{mg} / \mathrm{mL}, 1: 200$ dilution; Santa Cruz Biotechnology, USA). Sections stained with FITC IgG 
anti-goat antibody served as negative controls. Sections were mounted with Vectashield medium and were visualized with an Axioscope 2 Plus fluorescence microscope (Carl Zeiss).

2.7. RT-PCR. The popliteal lymph node was homogenized using the TH-01 (OMNI-INC). The total RNA from the lymph node and aortic valve was extracted using the TRIzol reagent according to the manufacturer's protocol. The reverse transcription was performed using $2 \mu \mathrm{g}$ of the total RNA, $200 \mathrm{U}$ of the reverse transcriptase, $2.5 \mu \mathrm{L}$ of the $5 \mathrm{X}$ RT buffer, $1.8 \mu \mathrm{L}$ of the $10 \mathrm{mM}$ dNTPs, $0.2 \mu \mathrm{L}$ of the $10000 \mathrm{U} / \mathrm{mL}$ RNasin, and $1.0 \mu \mathrm{L}$ of the $50 \mu \mathrm{M}$ oligo dT. The temperature settings for this reaction were $70^{\circ} \mathrm{C}$ for $5 \mathrm{~min}$, on ice for $2 \mathrm{~min}, 42^{\circ} \mathrm{C}$ for $60 \mathrm{~min}, 70^{\circ} \mathrm{C}$ for $15 \mathrm{~min}$, and $4^{\circ} \mathrm{C}$ for the final step. The resulting cDNA was used for real-time PCR as described later. The specific primers were designed using the Primer Express software and synthesized by IDT. Real-time PCR was carried out on a StepOne sequence detection system (Applied Biosystems) using the Power SYBR Green PCR Master Mix (Applied Biosystems). The dissociation curve indicated that only one product was obtained in each reaction. The relative levels of gene expression were determined using the $\Delta \Delta$ cycle threshold method as described by the manufacturer, in which data for each sample is normalized to the $\beta$-actin expression and the data are shown as the relative expression (the fold increase over the control). The PCR results were analyzed with the SDS 2.1 software (Applied Biosystems), and the amount of mRNA of each gene of interest was normalized to the amount of the murine $\beta$-actin gene. mRNA expression levels were calculated as the fold difference relative to the housekeeping gene by the formula: relative expression $=$ $2^{-(\text {CT }[\text { target gene]-CT }[\beta \text {-actin-1]). }}$

The sequences of the primers used are as follows:

VCAM-1: $\quad$ 5'-CCCCAAGGATCCAGAGATTCA-3' and $5^{\prime}$-ACTTGACCGTGACCGGCTT-3';

CD36: 5'GTACAGATTTGTTCTTCCAGCCAAT-3' and $5^{\prime}$-TCAGTGCAGAAACAATGGTTGTC- $3^{\prime}$;

MCP1/CCL2: 5'-AGGAAGATCTCAGTGCAGAG$3^{\prime}$ and $5^{\prime}$-AGTCTTCGGAGTTTGCCTTTG-3';

$\beta$-actin: $5^{\prime}$-CTGCCTGACGGCCAAGTC- $3^{\prime}$ and $5^{\prime}$ CAAGAAGGAA GGCTGGAAAAGA- $3^{\prime}$;

IL-6: 5'-ACAACCACGGCCTTCCCTACTT3' and $5^{\prime}$-CACGATTTCCCAGAGAACATGTG- ${ }^{\prime}$;

IFN- $\gamma: 5^{\prime}$-TGGCTCTGCAGGATTTTCATG- $3^{\prime}$ and $5^{\prime}$-TCAAGTGGCATAGATGTGGAAGAA- $3^{\prime}$;

IL-10: $5^{\prime}$-GGTTGCCAAGCCTTATCGGA- ${ }^{\prime}$ and $5^{\prime}$ ACCTGCTCCACTGCCTTGCT-3';

IL-4: $5^{\prime}$-ACAGGAGAAGGGACGCCAT- $3^{\prime}$ and $5^{\prime}$ GAAGCCCTACAGACGAGCTCA-3';

LmjF36.5520: 5 '-GGCCATCACCACAAACAGAG$3^{\prime}$ and 5-GCTCAGGTCATACAAGGGGA-3'.

2.8. Statistical Analyses. The data were initially analyzed by the Kolmogorov-Smirnov test to check their symmetry and by the Mann-Whitney or Student's $t$-test using GraphPad
TABLE 1: Final body weight, food intake, and cecal and hepatic lipids of non-infected (control) and L. major-infected (L. major) apoE KO mice 6 weeks after the inoculation of $1 \times 10^{6}$ parasites (clone WHO MHOM/IL/80/Friedlin) into footpad.

\begin{tabular}{lcc}
\hline Parameter & Control ApoE KO & L. major ApoE KO \\
\hline Final body weight (g) & $22.18 \pm 0.77$ & $20.67 \pm 0.7$ \\
Food intake (g/week/mouse) & $25.10 \pm 0.95$ & $24.72 \pm 0.85$ \\
Liver lipids & & \\
$\quad$ Total lipids (mg/g) & $252.0 \pm 12.0$ & $172.0 \pm 21.0^{*}$ \\
$\quad$ Total cholesterol (mg/g) & $14.1 \pm 1.3$ & $13.2 \pm 0.9$ \\
$\quad$ Triacylglycerols (mg/g) & $49.7 \pm 4.4$ & $30.2 \pm 4.5^{*}$ \\
Cecum lipids & & \\
Total lipids (mg/g) & $51.1 \pm 8.9$ & $70.0 \pm 11$ \\
Total cholesterol (mg/g) & $2.8 \pm 0.3$ & $2.4 \pm 0.3$ \\
Triacylglycerols (mg/g) & $0.97 \pm 0.2$ & $0.86 \pm 0.21$ \\
\hline
\end{tabular}

Control group, $n=8-11$, and L. major group, $n=10-13$. Results are expressed as the means \pm SE. ${ }^{*} P \leq 0.05$.

Prism, version 5.00, for Windows, GraphPad Software, San Diego, CA, USA. A $P$ value of $<0.05$ was considered significant.

\section{Results}

3.1. L. major Infection in ApoE KO and Wild-Type Control C57BL/6 Mice. Our first aim was to confirm that L. major causes a self-limited infection in apoE $\mathrm{KO}$ mice similar to that observed in the wild-type control C57BL/6 mice. The results showed that after 6 weeks of infection, although apoE KO mice presented higher levels of the cytokines TNF alpha and IL-10 (Figures 1(a) to 1(d)), these mice controlled parasite load and footpad swelling similarly to C57BL/6 mice (Figures 1(e) and $1(\mathrm{f})$ ). Therefore, only apoE KO animals were used for further experiments.

There were no differences in the food intake between apoE experimental and control groups. As expected, L. major infection did not cause a significant weight loss in these animals (Table 1).

3.2. Lipid Profile. L. major infection was associated with increased serum cholesterol due to an increase in both HDL and non-HDL fractions (Figure 2). These alterations in the lipid profile were not due to differences in the hepatic cholesterol content or cecal excretion because the levels of these were similar between groups (Table 1).

L. major infection resulted in a reduction in the blood and hepatic triacylglycerol content compared to the controls (Figure 2(a) and Table 1), highlighting the possible influence of L. major on triacylglycerol metabolism. With regard to blood cholesterol, L. major infection was associated with increased serum total cholesterol due to an increase in both HDL and non-HDL fractions (Figures 2(b) to 2(d)), highlighting the influence of this parasite also on plasma cholesterol levels. 


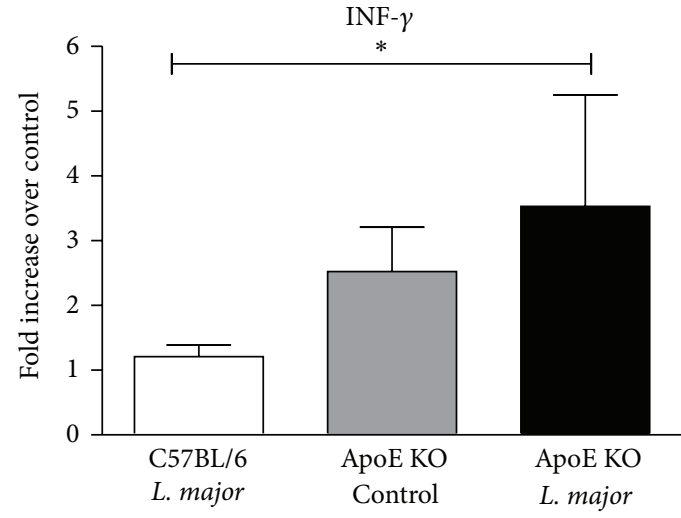

(a)

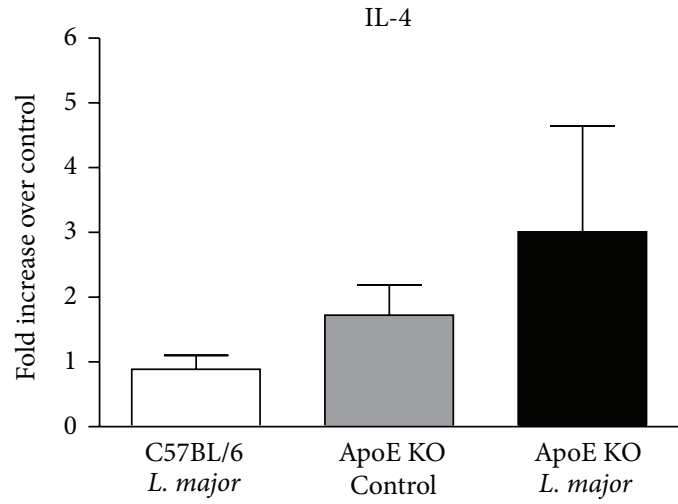

(c)

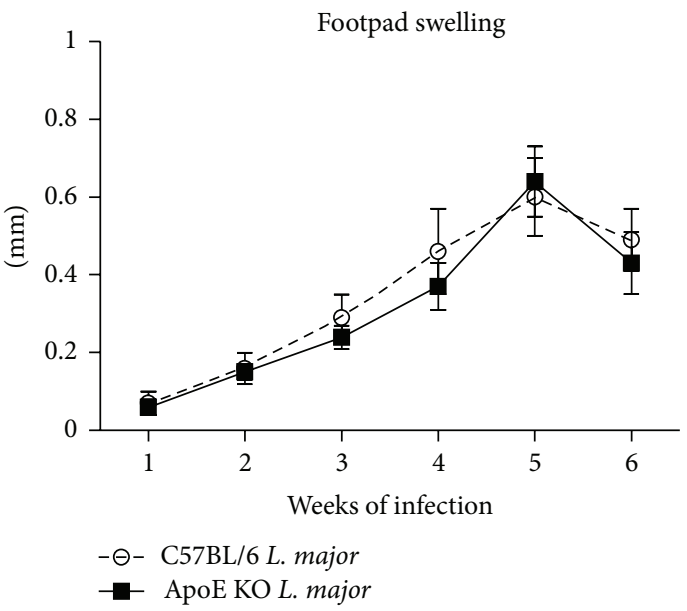

(e)

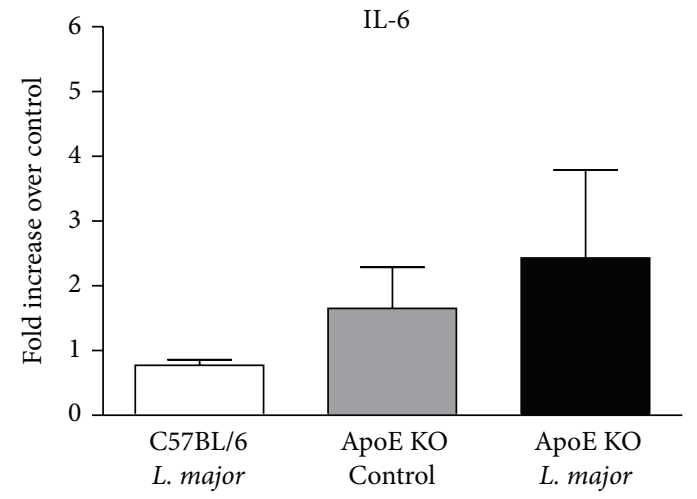

(b)

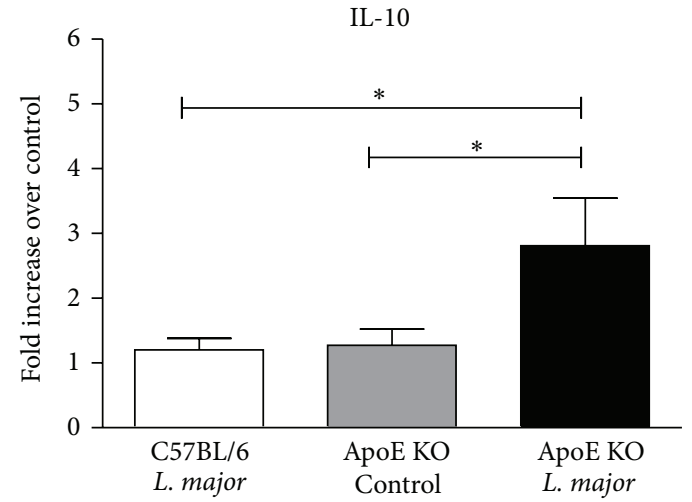

(d)

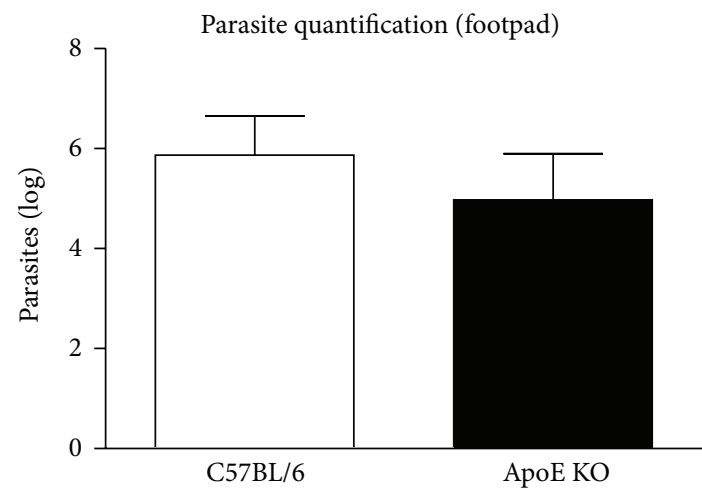

FIGURE 1: The infection profile of L. major in apoE $\mathrm{KO}$ and C57BL/6 animals infected with $1 \times 10^{6}$ parasites (clone WHO MHOM/IL/80/Friedlin) into left footpad. (a to d) Cytokine (IL-4, IL-10, TNF- $\alpha$, and IL-6) mRNA transcripts in the popliteal lymph nodes of apoE KO control, apoE KO L. major-infected, and C57BL/6 L. major-infected ( $n=4 /$ group) mice at the 6th experimental week. Values of average and standard error of cytokine mRNA transcripts in C57BL infected, apoE KO control, and apoE KO infected were, respectively, $\mathrm{IL}-4=0.89 \pm 0.20 ; 1.72 \pm 0.46$ and $3.01 \pm 1.6 ; \mathrm{IL}-10=1.2 \pm 0.12 ; 1.28 \pm 0.24$ and $2.81 \pm 0.7 ;$ IFN $\gamma=1.20+0.82 ; 2.53 \pm 0.63$ and $3.52+1.7 ; \mathrm{IL}-6=$ $0.78 \pm 0.80 ; 1.65 \pm 0.63$ and $2.4 \pm 1.3$. (e) The infection curve of apoE KO and C57BL/6 L. major infected mice. The results are expressed as the average of the footpad swelling calculated by the difference between the measures of the infected (left) and control (right) footpads. Number of mice: $n=11$ in apoE KO and $n=7$ in C57BL/6 groups for each week. (f) Footpad parasite quantification from apoE KO and C57BL/6 mice infected with L. major 6 weeks after infection. The results are expressed as the average of the log of the total parasites from apoE KO $(n=11)$ and C57BL/6 $(n=7)$ mice. Bars = average and vertical lines $=$ standard error. ${ }^{*} P<0.05$. 


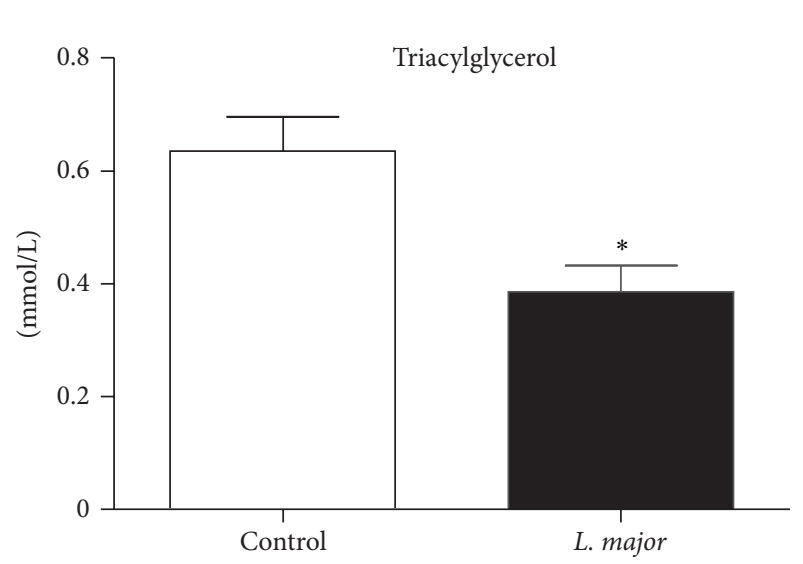

(a)

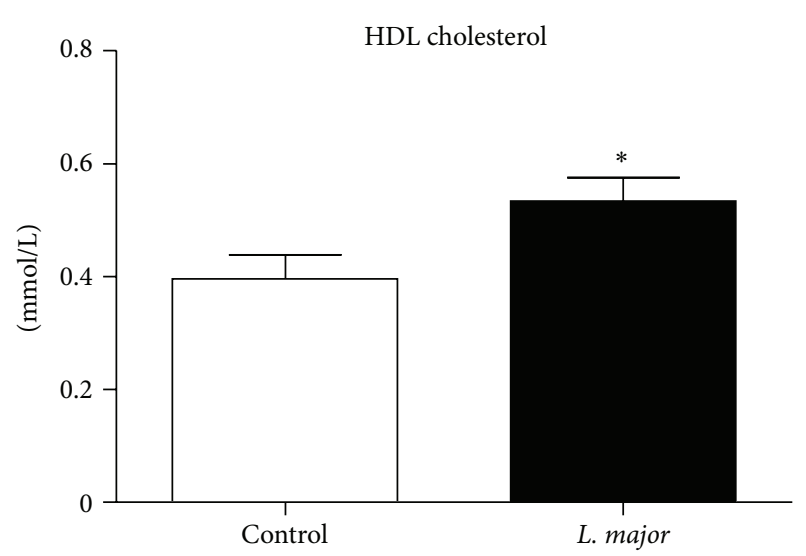

(c)

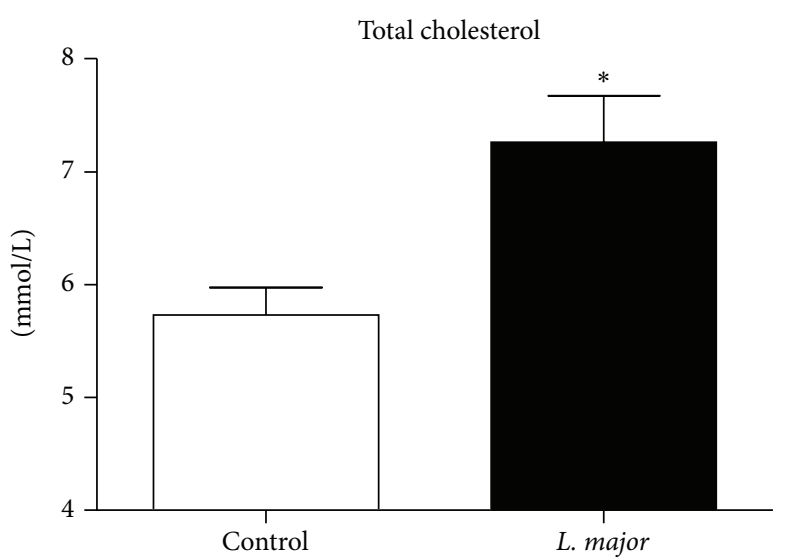

(b)

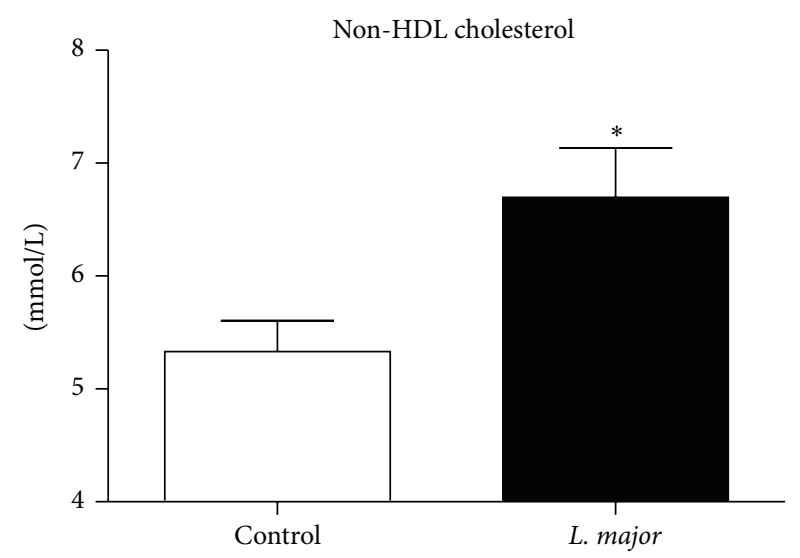

(d)

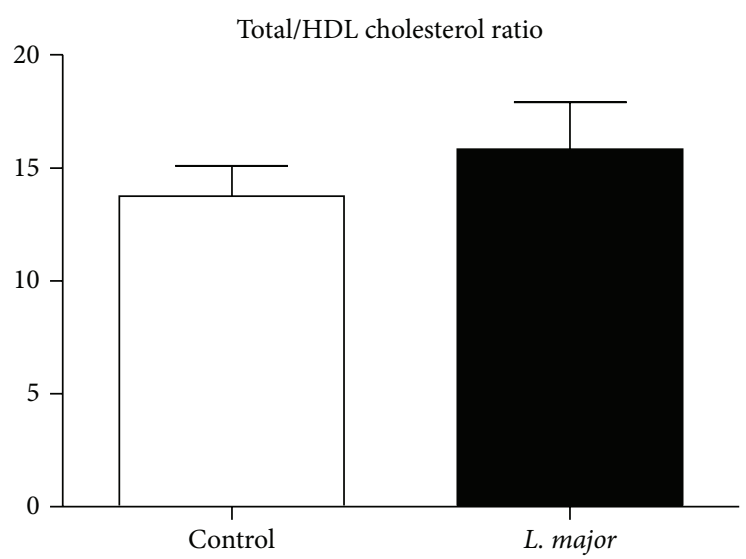

(e)

FIGURE 2: Blood lipid profile of noninfected $(n=9)$ and L. major-infected $(n=12)$ apoE KO mice 6 weeks after the inoculation of $1 \times 10^{6}$ parasites (clone WHO MHOM/IL/80/Friedlin) into left footpad. (a) Total triacylglycerols; (b) total cholesterol; (c) high density lipoprotein (HDL) cholesterol; and (d) non-HDL cholesterol (sum of cholesterol in low density (LDL), intermediate density (IDL) lipoproteins, and remnant chylomicron), calculated as the difference between cholesterol and HDL cholesterol. Bars = average and vertical lines $=$ standard error. ${ }^{*} P<0.05$.

3.3. Atherosclerotic Lesions. Animals infected with L. major presented larger and more developed atherosclerotic lesions when compared to the controls (Figures 3(a), 3(c), and 3(e)). In the control group, atherosclerotic lesions were formed by fatty streaks and several layers of foam cells. On the other hand, in the infected animals, an intense inflammatory infiltrate and necrotic areas with deposition of cholesterol crystals (characteristics of an advanced atherosclerotic lesion) were frequently found (Figures 3(b), 3(d), and 3(f)). The inflammatory infiltrate was composed by cells of the innate immune 


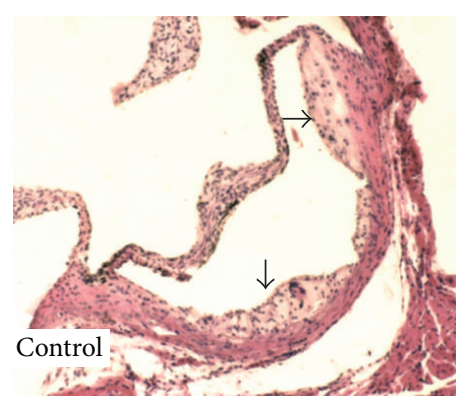

(a)

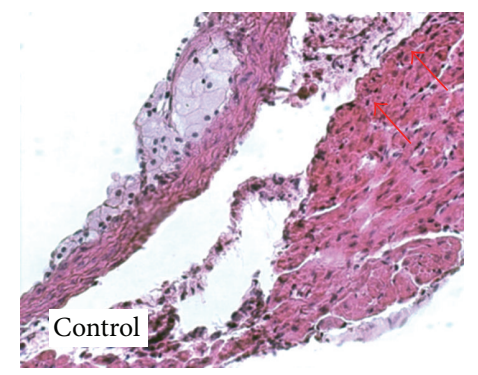

(d)

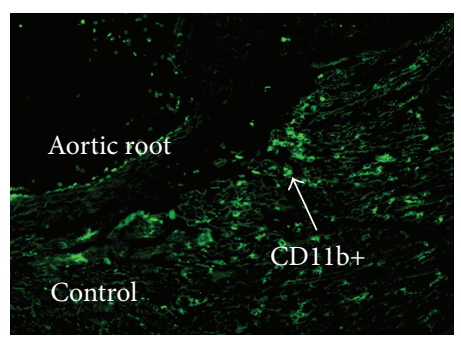

(g)

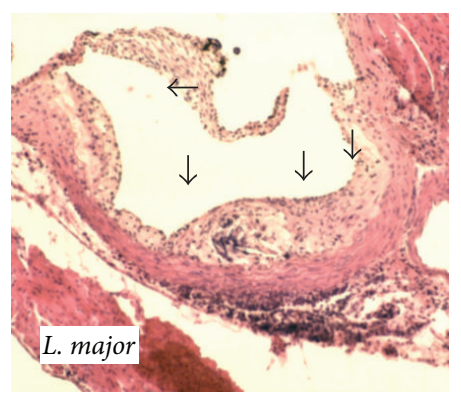

(b)

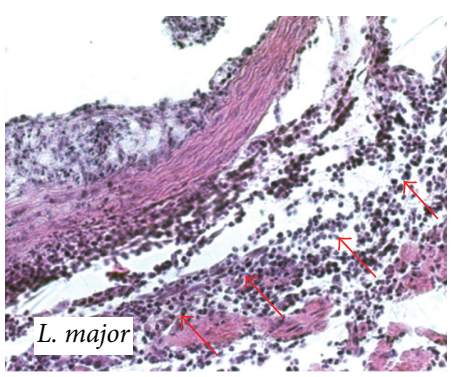

(e)

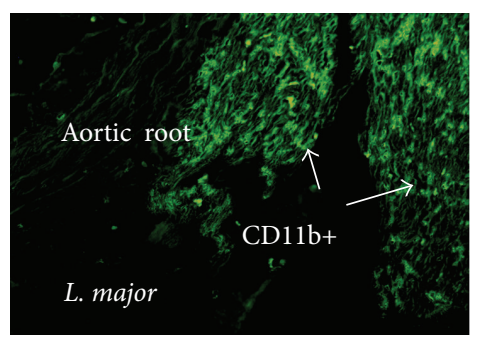

(h)

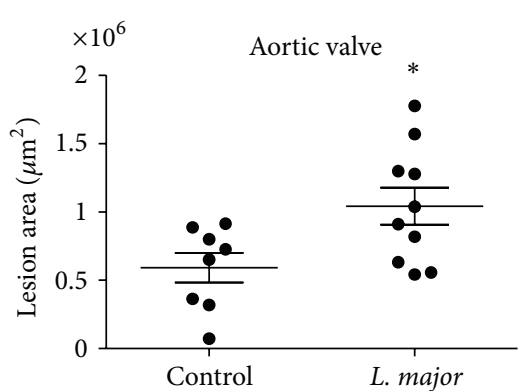

(c)

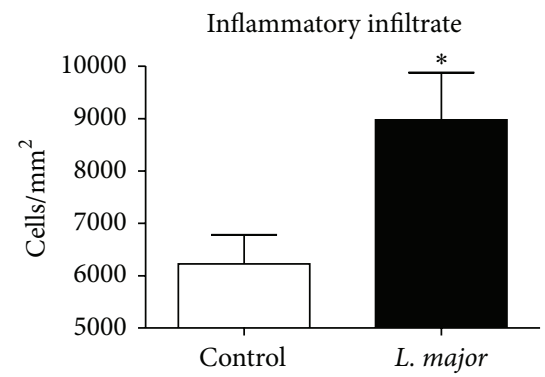

(f)

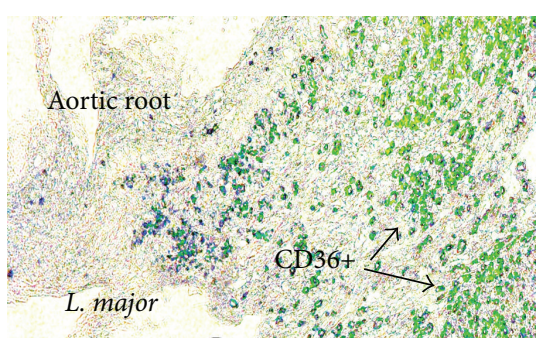

(i)

Figure 3: Atherosclerotic lesions and inflammatory infiltrate in apoE KO mice infected or noninfected (control) with $L$. major for 6 weeks. (a, b) Histology of the aorta from noninfected (a) and L. major-infected (b) apoE KO mice, 6 weeks after infection. (c) Atherosclerosis lesion area in aortic valve of noninfected (Control) and L. major-infected apoE KO mice. The results represent the average of the lesion area $\left(\mu \mathrm{m}^{2}\right)$ in the aortic valve of animal from the control $(n=7)$ and the infected groups $(n=10), 6$ weeks after infection. The major lines represent the means and the minor lines represent the standard error. ${ }^{*} P=0.05$. (d, e) Inflammatory infiltrate around the atherosclerotic lesion in aortic valve of non-infected (d) and L. major-infected (e) apoE KO mice. (f) Average number of cells $/ \mathrm{mm}^{2}$ of aortic valve of non-infected $(n=5)$ and infected $(n=5)$ apoE KO mice 6 weeks after infection. The bars represent the average and vertical lines represent standard error, ${ }^{*} \mathrm{P}<$ 0.05. (g, h) Immunofluorescence: CD11b FITC conjugated positive cells in aortic root of control (g) and L. major-infected (h) mice. (i) Anti-CD36 antibody Alexa488 conjugated in aortic valve of L. major-infected group. In (a), (b), (d), and (e), the sections were stained with H\&E. Image magnification: 20x in (a), (b), (g), and (h) and 100x in (d), (e), and (i).

system, including monocytes and macrophages as suggested by the higher number of CD11b positive cells around the atherosclerotic lesion of infected mice (Figures $3(\mathrm{~g})$ and $3(\mathrm{~h})$ ).

3.4. Expression of VCAM-1, MCP1/CCL2, and CD36 in the Aortic Valve. The expression of the adhesion molecule VCAM-1, the monocyte chemotactic protein MCP-1/CCL2, and the scavenger receptor CD36 was also assessed in atherosclerotic lesions.

Confirming the histological data, the results showed that CD36 expression was significantly increased in the L. major group $(P=0.02)$. There was a tendency for increased $(P=$ $0.11) \mathrm{MCP} 1 / \mathrm{CCL} 2$ expression, and there were no changes in VCAM $1(P=0.67)$ expression (Figure 4$)$.
3.5. L. major DNA in Peripheral Tissues. Spleen and liver were tested for the presence of L. major DNA. The parasite was not detected in the spleen or liver of mice from both groups, suggesting the absence of viable parasites in these organs (Figure 5).

\section{Discussion}

To our knowledge, this is the first study investigating the effect of Leishmania infection on atherogenesis. A previous study [21] using apoE KO mice analyzed the influence of genetic and high-fat/cholesterol diet-induced dyslipidemia on $\mathrm{T}$ cell and dendritic cell (DC) responses to L. major infection in vivo and, mainly, in vitro. Although using the same mouse 


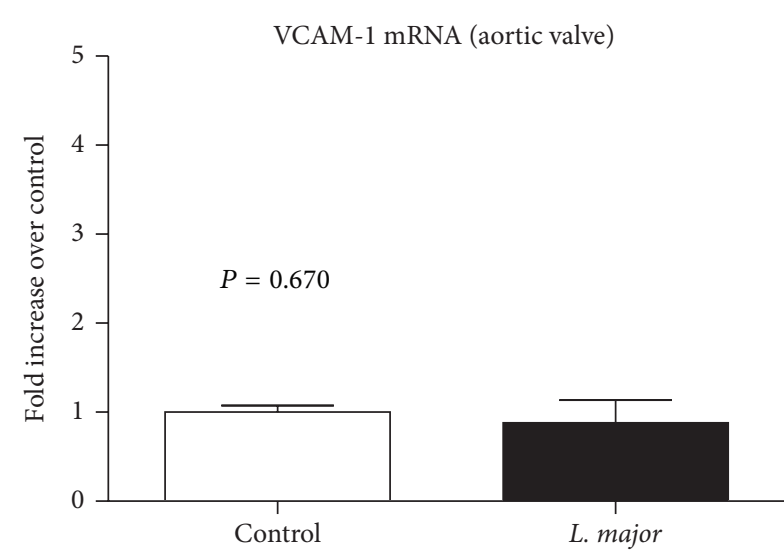

(a)

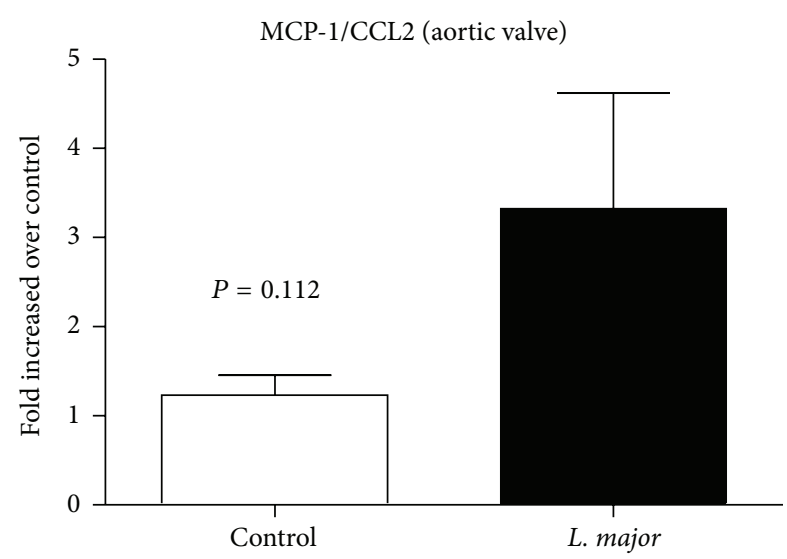

(b)

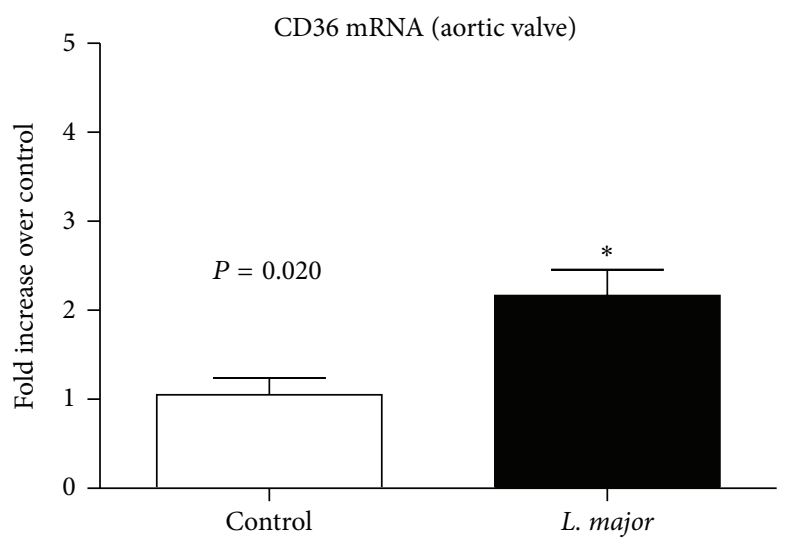

(c)

FIGURE 4: Relative increase of (a) VCAM-1, (b) MCP1/CCL2, and (c) CD36RNA expression in the aortic valve of non-infected and infected apoE KO mice, 6 weeks after infection. Bars = average and vertical lines $=$ standard error, $n=4$ /group. ${ }^{*} P<0.05$. The values of apoE KO control and $L$. major groups were, respectively, $\mathrm{VCAM}=1.01 \pm 0.07$ and $0.89 \pm 0.21 ; \mathrm{CD} 36=1.05 \pm 0.19$ and $2.16 \pm 0.20 ; \mathrm{MCP}-1=1.22 \pm 0.22$ and $2.75 \pm 0.83$.
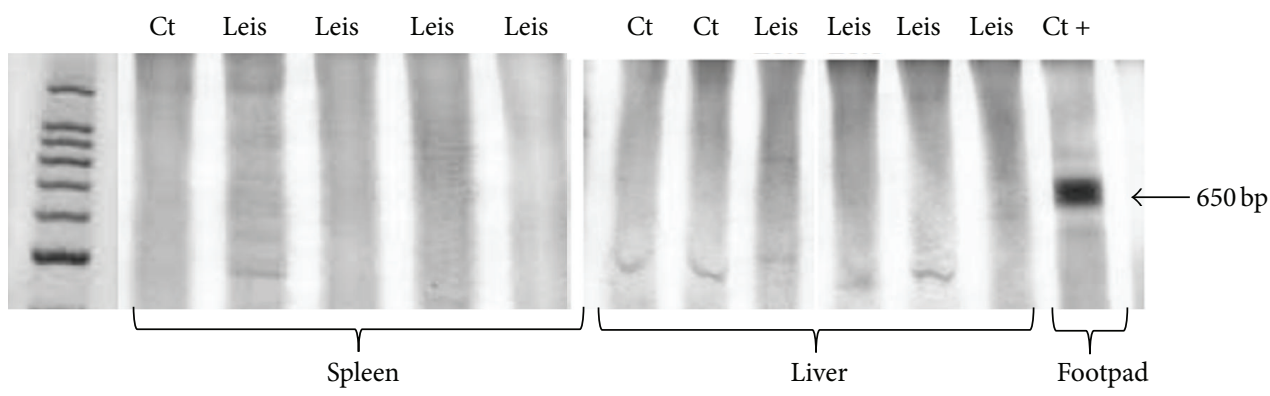

FIGURE 5: PCR amplification of L. major DNA in the spleen and liver of non-infected and L. major-infected apoE KO mice. First line: molecular weight marker; last line: positive control DNA from an L. major-infected footpad. Ct = apoE KO control mice; Leis- apoE KO L. major-infected mice.

model used in the present study, the authors did not analyze atherosclerosis development and lipid profile. Moreover, cytokine determinations were done mainly in vitro, after parasite antigen restimulation. Finally, the resolution of $L$. major infection in C57BL/6 mice (measured by footpad swelling) started only after 10 weeks of infection while in ours and other studies [22-24] it occurred after 5 weeks of infection.
Our results show that L. major infection modifies blood lipid metabolism, resulting in the increase of total cholesterol and its fractions. In addition to the hypercholesterolemia, L. major infection also interfered with the metabolism of triacylglycerols, reducing their levels in the blood and liver. Despite the reduction in blood triacylglycerols, we found larger and more advanced atherosclerotic lesions in the aortic valves of infected mice. 
Our previous studies showed that T. gondii, which induces a strong systemic inflammation, promotes atherogenesis in apoE KO mice [11]. Because L. major infection is self-limited in mice, we expected that L. major would have little influence on atherosclerosis development compared to T. gondii infection. However, our results suggest an important role for $L$. major infection in macrophage activation and atherosclerosis development.

Several mouse strains, including C57BL/6, effectively control L. major infection, while others, such as the BALB/c strain, develop progressive damage to the site of infection and systemic disease [25]. Our results indicate that apoE KO animals present the same pattern of resistance to $L$. major infection as that observed in the C57BL/6 animals, as determined by similar degrees of parasite load and footpad swelling. However, the pattern of cytokine expression after 6 weeks of infection shows a strong tendency to be higher in ApoE KO infected mice compared to the ApoE control and the C57BL/6 infected groups. This higher inflammatory status of ApoE $\mathrm{KO}$ mice has been previously presented as a consequence of the absence of anti-inflammatory effects of apoE as well as the proinflammatory stimulus of hypercholesterolemia and oxidized lipoproteins [26].

L. major infection leads to hypercholesterolemia associated with a reduction in blood and hepatic triacylglycerols without changes in cholesterol excretion. This pattern of lipid alteration was not found during a T. gondii infection [11], which reduces cholesterolemia with no alterations in the level of triacylglycerols in the blood. These different findings reflect the specific metabolic characteristics of each protozoan with regard to their use of host lipids. T. gondii is unable to produce cholesterol on its own. Thus, it is dependent on host cholesterol taken from the blood. Additionally, $T$. gondii causes a more severe infection that induces a reduction in food intake and weight loss in the mice. One of the consequences of this is the preferential use of acetyl CoA to supply energy, reducing its availability to the cholesterol synthesis pathway [27]. Both factors contribute (host cholesterol uptake and acetyl-coA redirection) to the reduction of cholesterolemia during a T. gondii infection. Leishmania, on the other hand, is able to synthesize its own cholesterol [28] but is dependent on host fatty acids, an important source of energy for amastigotes [29]. It is possible that L. major acquires fatty acids from triacylglycerol-rich lipoproteins, particularly IDL, which is the primary lipoprotein in apoE KO mice, resulting in the decrease of circulating triacylglycerol from lipoproteins in infected mice. On the other hand, the reduction of triacylglycerol could be related to the weight loss and a catabolic state. Independently of the cause, the reduction of the blood triacylglycerols stimulates the rapid conversion of the IDL into the more atherogenic, small, and dense LDL [30], which could explain the higher cholesterol and atherogenic fractions in the L. major group [31, 32].

L. major infection also stimulated the migration of inflammatory cells to the atherosclerotic lesion site. Aside from the hypercholesterolemia, these results could be explained by the oxidative stress and endothelial dysfunction resulting from inflammation [33], which leads to rapid migration of immune cells to the atherosclerotic lesion, where they accelerate atherogenesis. Moreover, L. major infected leukocytes could egress from the footpad tissue or draining lymph node and, following chemotactic stimulus, and migrate to other inflammatory regions such as the atherosclerotic site. Macrophages are connected to the progression of atherosclerosis through the production of inflammatory cytokines, which activate endothelial mediators and prothrombotic factors that are important in the atherosclerosis development. The increased movement of L. major-activated leukocytes to the site of atherosclerosis is suggested by the higher inflammatory infiltration and the higher expression of CD36, the major macrophage scavenger receptor for abnormal (oxidized) LDL. The introduction of minimally oxidized LDL into the intimae of arteries causes endothelial activation and release of leukocyte chemotactic factors that attract macrophages and other immune cells, which aggravates the inflammation and enhances plaque formation. In our study, parasite DNA was not found in the spleen or liver, refuting the hypothesis of visceralization.

In conclusion, L. major infection, although localized and self-limited in resistant apoE KO mice, has a detrimental effect on the blood lipid profile, increasing the inflammatory cell migration to atherosclerotic lesions and accelerating atherogenesis. The latter is the consequence of the increase of the inflammatory component of atherosclerosis that could be trigged by the parasite-activated macrophages.

\section{Acknowledgments}

This work was supported by PRPq/UFMG, Pró-reitoria de Pesquisa of Universidade Federal de Minas Gerais, CNPq (Conselho Nacional de Desenvolvimento Científico e Tecnológico), and CAPES (CAPES-Coordenação de Aperfeiçoamento de Pessoal de Nível. Superior). The authors are grateful to Maria Helena Alves de Oliveira, who was responsible for the animal facility. This work was conducted at the Universidade Federal de Minas Gerais, Brazil.

\section{References}

[1] J. Liese, U. Schleicher, and C. Bogdan, "The innate immune response against Leishmania parasites," Immunobiology, vol. 213, no. 3-4, pp. 377-387, 2008.

[2] G. K. Hansson, "Inflammatory mechanisms in atherosclerosis," Journal of Thrombosis and Haemostasis, vol. 7, no. 1, pp. 328-331, 2009.

[3] R. Ross, "Atherosclerosis-an inflammatory disease," The New England Journal of Medicine, vol. 340, no. 2, pp. 115-126, 1999.

[4] G. K. Hansson and P. Libby, "The immune response in atherosclerosis: a double-edged sword," Nature Reviews Immunology, vol. 6, no. 7, pp. 508-519, 2006.

[5] S. Kiechl, G. Egger, M. Mayr et al., "Chronic infections and the risk of carotid atherosclerosis: prospective results from a large population study," Circulation, vol. 103, no. 8, pp. 1064-1070, 2001.

[6] G. Noll, "Pathogenesis of atherosclerosis: a possible relation to infection," Atherosclerosis, vol. 140, no. 1, pp. S3-S9, 1998. 
[7] F. F. Mussa, H. Chai, X. Wang, Q. Yao, A. B. Lumsden, and C. Chen, "Chlamydia pneumoniae and vascular disease: an update," Journal of Vascular Surgery, vol. 43, no. 6, pp. 1301-1307, 2006.

[8] L. J. Murray, K. B. Bamford, D. P. J. O’Reilly, E. E. McCrum, and A. E. Evans, "Helicobacter pylori infection: relation with cardiovascular risk factors, ischaemic heart disease, and social class," British Heart Journal, vol. 74, no. 5, pp. 497-501, 1995.

[9] M. S. Burnett, S. Durrani, E. Stabile et al., "Murine cytomegalovirus infection increases aortic expression of proatherosclerotic genes," Circulation, vol. 109, no. 7, pp. 893-897, 2004.

[10] M. J. Doenhoff, R. G. Stanley, K. Griffiths, and C. L. Jackson, "An anti-atherogenic effect of Schistosoma mansoni infections in mice associated with a parasite-induced lowering of blood total cholesterol," Parasitology, vol. 125, no. 5, pp. 415-421, 2002.

[11] L. R. Portugal, L. R. Fernandes, G. C. Cesar et al., "Infection with Toxoplasma gondii increases atherosclerotic lesion in ApoEdeficient mice," Infection and Immunity, vol. 72, no. 6, pp. 35713576, 2004.

[12] J. H. Meurman, M. Sanz, and S. J. Janket, "Oral health, atherosclerosis, and cardiovascular disease," Critical Reviews in Oral Biology and Medicine, vol. 15, no. 6, pp. 403-413, 2004.

[13] H. Zhang, L. M. Wu, and J. Wu, "Cross-talk between apolipoprotein E and cytokines," Mediators of Inflammation, vol. 2011, Article ID 949072, 10 pages, 2011.

[14] L. S. Capettini, S. F. Cortes, J. F. Silva, J. I. Alvarez-Leite, and V.S. Lemos, "Decreased production of nNOS-derived hydrogen peroxide contributes to endothelial dysfunction in atherosclerosis," British Journal of Pharmacology, vol. 164, no. 6, pp. 17381748, 2011.

[15] P. G. Reeves, F. H. Nielsen, and G. C. Fahey, "AIN-93 purified diets for laboratory rodents: final report of the american institute of nutrition ad hoc writing committee on the reformulation of the AIN-76A rodent diet," The Journal of Nutrition, vol. 123, no. 11, pp. 1939-1951, 1993.

[16] L. Q. Vieira, M. Goldschmidt, M. Nashleanas, K. Pfeffer, T. Mak, and P. Scott, "Mice lacking the TNF receptor p55 fail to resolve lesions caused by infection with Leishmania major, but control parasite replication," The Journal of Immunology, vol. 157, no. 2, pp. 827-835, 1996.

[17] S. Fazio, V. R. Babaev, A. B. Murray et al., "Increased atherosclerosis in mice reconstituted with apolipoprotein $\mathrm{E}$ null macrophages," Proceedings of the National Academy of Sciences of the United States of America, vol. 94, no. 9, pp. 4647-4652, 1997.

[18] J. Folch, M. Lees, and S. G. H. Sloane, "A simple method for the isolation and purification of total lipides from animal tissues," The Journal of biological chemistry, vol. 226, no. 1, pp. 497-509, 1957.

[19] B. Paigen, A. Morrow, P. A. Holmes, D. Mitchell, and R. A. Williams, "Quantitative assessment of atherosclerotic lesions in mice," Atherosclerosis, vol. 68, no. 3, pp. 231-240, 1987.

[20] G. Anders, C. L. Eisenberger, F. Jonas, and C. L. Greenblatt, "Distinguishing Leishmania tropica and Leishmania major in the Middle East using the polymerase chain reaction with kinetoplast DNA-specific primers," Transactions of the Royal Society of Tropical Medicine and Hygiene, vol. 96, supplement 1, pp. S87-S92, 2002.

[21] A. T. Shamshiev, F. Ampenberger, B. Ernst, L. Rohrer, B. J. Marsland, and M. Kopf, "Dyslipidemia inhibits Toll-like receptor-induced activation of CD8 $\alpha$-negative dendritic cells and protective Th1 type immunity," Journal of Experimental Medicine, vol. 204, no. 2, pp. 441-452, 2007.

[22] H. C. Santiago, C. F. Oliveira, L. Santiago et al., "Involvement of the chemokine RANTES (CCL5) in resistance to experimental infection with Leishmania major," Infection and Immunity, vol. 72, no. 8, pp. 4918-4923, 2004.

[23] C. F. Oliveira, D. Manzoni-de-Almeida, P. S. Mello et al. , "Characterization of chronic cutaneous lesions from TNF-receptor1-deficient mice infected by Leishmania major," Clinical and Developmental Immunology, vol. 2012, Article ID 865708, 12 pages, 2012.

[24] F. Benhnini, M. Chenik, D. Laouini, H. Louzir, P. A. Cazenave, and K. Dellagi, "Comparative evaluation of two vaccine candidates against experimental leishmaniasis due to Leishmania major infection in four inbred mouse strains," Clinical and Vaccine Immunology, vol. 16, no. 11, pp. 1529-1537, 2009.

[25] D. Sacks and N. Noben-Trauth, "The immunology of susceptibility and resistance to Leishmania major in mice," Nature Reviews Immunology, vol. 2, no. 11, pp. 845-858, 2002.

[26] X. Zhou, G. Paulsson, S. Stemme, and G. K. Hansson, "Hypercholesterolemia is associated with a T helper (Th) 1/Th2 switch of the autoimmune response in atherosclerotic apo E-knockout mice," Journal of Clinical Investigation, vol. 101, no. 8, pp. 1717$1725,1998$.

[27] G. R. Jansen, M. E. Zanetti, and C. F. Hutchison, "Stdies on lipogenesis in vivo. Effects of starvation andre-feeding, and studies on cholesterol synthesis," Biochemical Journal, vol. 99, no. 2, pp. 333-340, 1966.

[28] M. L. Ginger, M. C. Prescott, D. G. Reynolds, M. L. Chance, and J. L. Goad, "Utilization of leucine and acetate as carbon sources for sterol and fatty acid biosynthesis by old and new world Leishmania species, Endotrypanum monterogeii and Trypanosoma cruzi," European Journal of Biochemistry, vol. 267, no. 9, pp. 2555-2566, 2000.

[29] D. T. Hart and G. H. Coombs, "Leishmania mexicana: energy metabolism of amastigotes and promastigotes," Experimental Parasitology, vol. 54, no. 3, pp. 397-409, 1982.

[30] M. Rizzo and K. Berneis, "Low-density lipoprotein size and cardiovascular risk assessment," QJM, vol. 99, no. 1, pp. 1-14, 2006.

[31] E. Esteve, W. Ricart, and J. M. Fernández-Real, "Dyslipidemia and inflammation: an evolutionary conserved mechanism," Clinical Nutrition, vol. 24, no. 1, pp. 16-31, 2005.

[32] C. G. Nieto, R. Barrera, M. A. Habela et al., "Changes in the plasma concentrations of lipids and lipoprotein fractions in dogs infected with Leishmania infantum," Veterinary Parasitology, vol. 44, no. 3-4, pp. 175-182, 1992.

[33] G. K. Hansson, "Inflammation, atherosclerosis, and coronary artery disease," The New England Journal of Medicine, vol. 352, no. 16, pp. 1685-1626, 2005. 


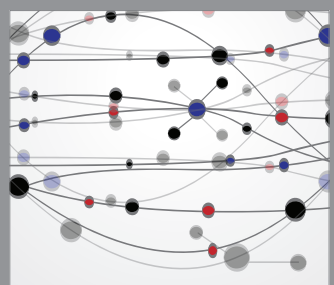

The Scientific World Journal
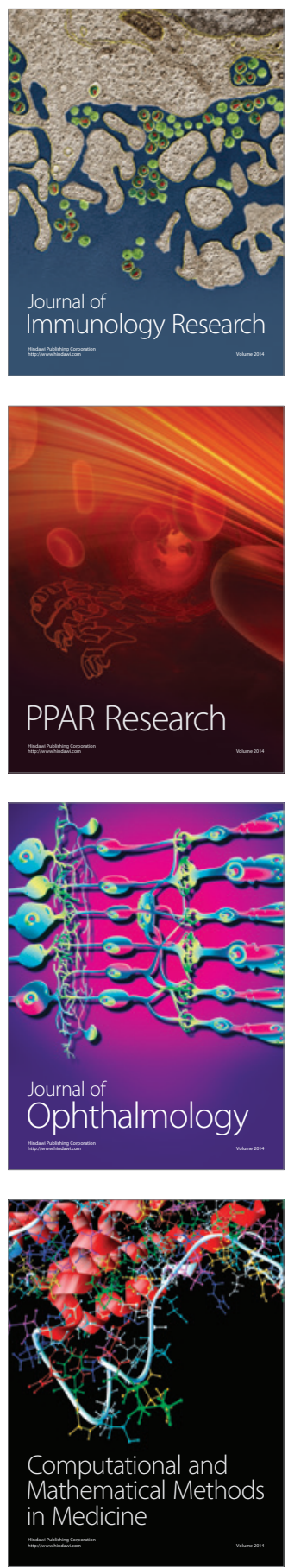

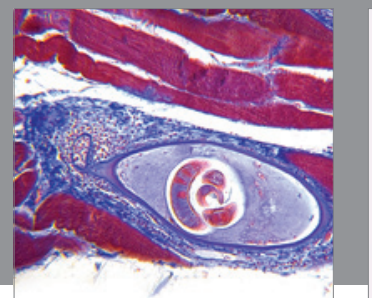

Gastroenterology

Research and Practice
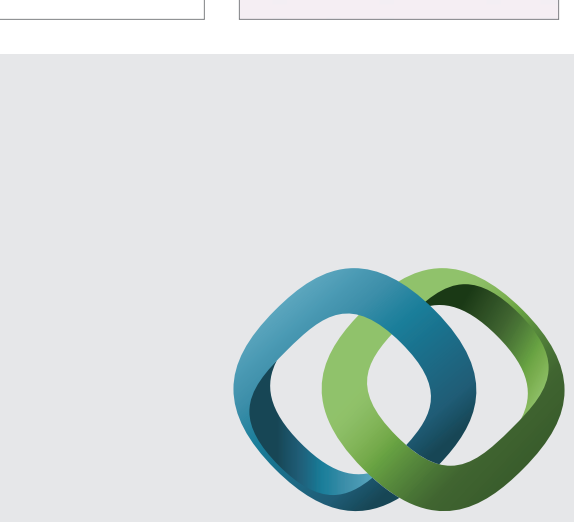

\section{Hindawi}

Submit your manuscripts at

http://www.hindawi.com
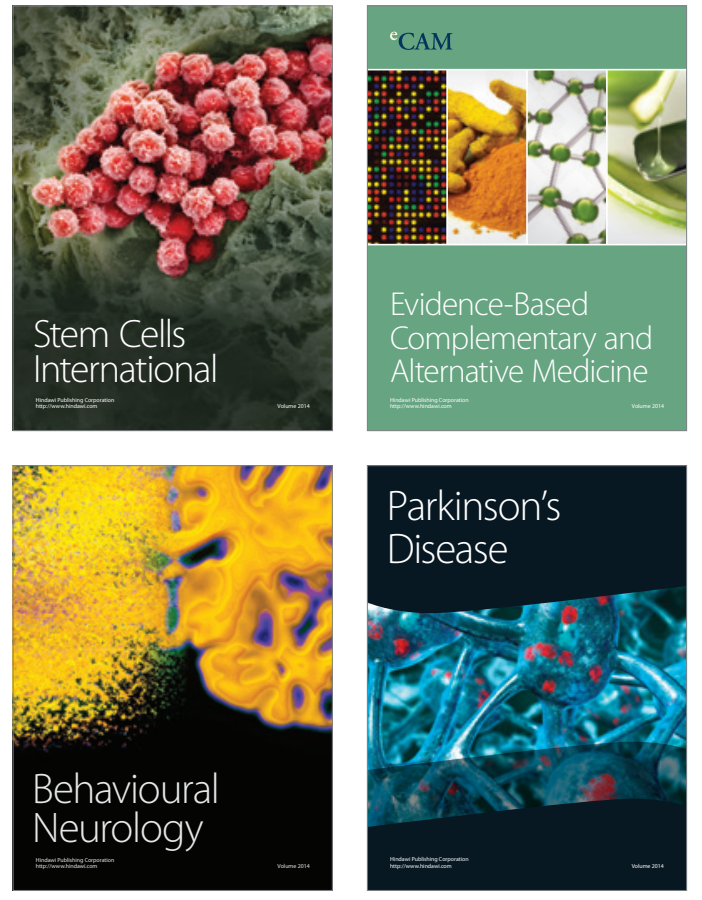
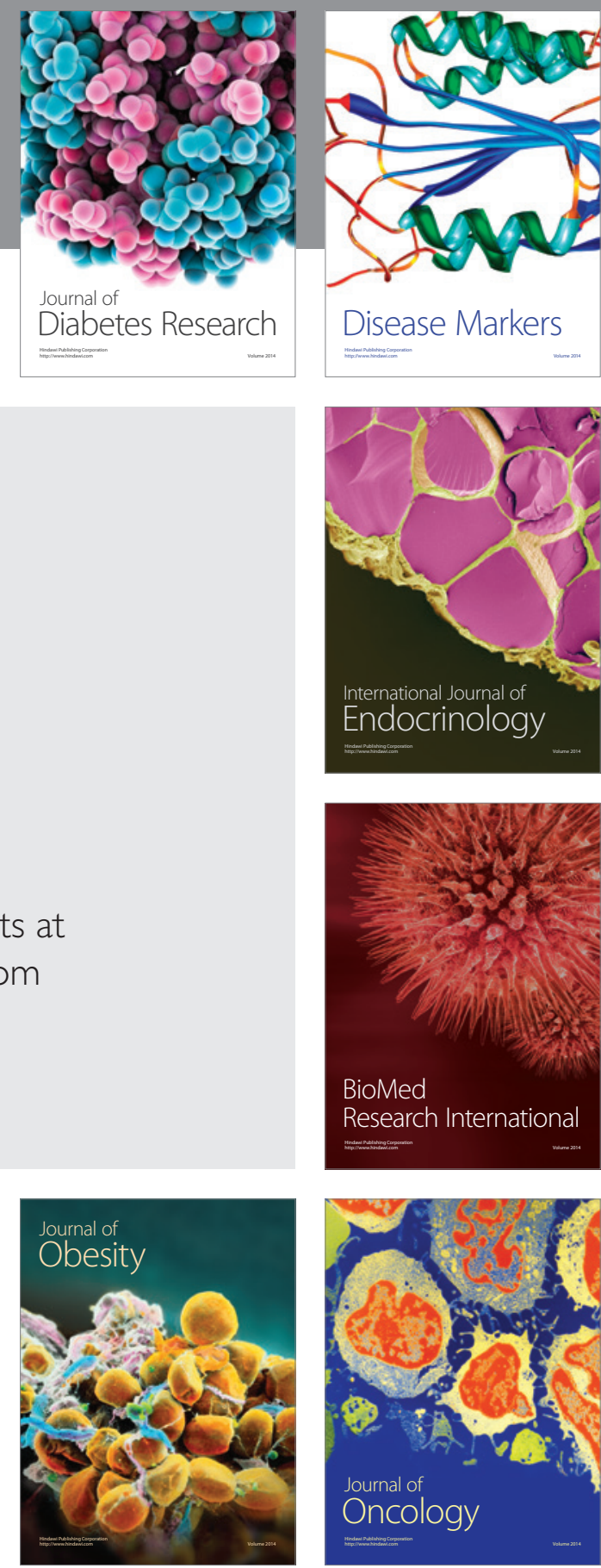

Disease Markers
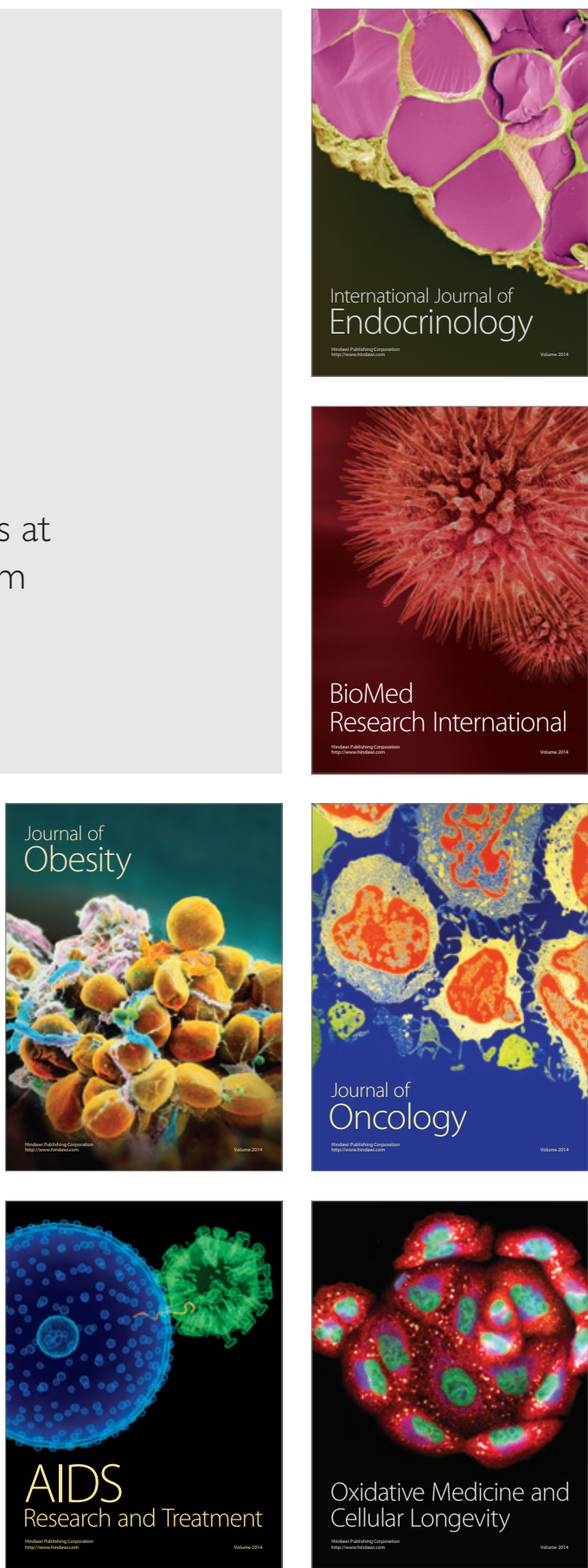